International Journal of

Advanced Science and Convergence

\title{
Used Trading platform with Hyperledger fabric
}

\author{
Sung-Yeon Cho ${ }^{1}$ and Sunghyuck Hong ${ }^{2}$ \\ ${ }^{1}$ Student, Division of ICT, Baekseok University, KOREA \\ ${ }^{2}$ Professor, Division of ICT, Baekseok University, KOREA
}

\begin{abstract}
Background/Objectives: Hyperledger fabric is a blockchain open source project hosted by the Linux foundation. The goal is to create blockchain technology that can be applied across industries such as finance, IoT, logistics, manufacturing and technology. Methods/Statistical analysis: In addition to Hyperledger, there are other blockchain platforms such as R3, Ripple, and Ethereum. The reason why Hyperledger is special is that: 1 ) it is a private blockchain platform that is suitable for realizing corporate business. The domestic secondary market is growing significantly. The domestic secondary market is over 2 trillion won annually. However, a number of criminal activities are taking place in the second transaction because of the inability to identify one another. Findings: To prevent damage, it is impossible to use the Hyperledger fabric to prove your identity and use chain code to forge post and transaction details. It also prevents fraud, duplicate transactions, counterfeiting and changes. In the case of criminal activity, the relevant nodes are checked to identify the offender's identity and then expedite processing. Improvements/Applications: For this feature, Hyperledger builds a highly reliable secondary trading platform with excellent confidentiality, resilience, flexibility and scalability and the ability to share trading records only with specific members of the network.
\end{abstract}

\section{Index Terms}

Blockchain, Used trade, responsibility, scalability, Hyperledger fabric

\section{INTRODUCTION}

The domestic secondhand market is growing rapidly with the development of the Internet. The size of the domestic used trading market is developing day by day, and its size is not estimated correctly, but it is estimated to be about 2 trillion won to 10 trillion won. However, a number of criminal acts are increasing in this huge market of secondhand trade. In order to

\footnotetext{
Corresponding author : Sunghyuck Hong

shong@bu.ac.kr

- Manuscript received July 10, 2019.

- Revised August 1, 2019 ; Accepted September 20, 2019.

- Date of publication September 30, 2019.
}

(C) The Academic Society of Convergence Science Inc.

2619-8150 @ 2019 IJASC. Personal use is permitted, but republication/redistribution requires IJASC permission. 
eliminate these criminal acts, we use blockchain that is difficult to forge. By using the non-forgery characteristic of the blockchain, mutual identity verification is assured, and the secondary damage is quickly responded to when damage occurs. In addition, in order to prevent financial and material damage in advance, the function of proactive prevention was created using Hyperledger fabric, a private blockchain platform. In addition, the function of storing the trader's IP and location in the used transaction is stored using Dynamo DB.

\section{HYPERLEDGER FABRIC}

\section{A. Hyperledger fabric}

Hyperledger fabric is a permission blockchain developed by the Linux foundation, which was established in 2015 to advance cross-industry blockchain technology. Instead of declaring a single blockchain standard, we encourage the development and access of blockchain technologies to community processes through intellectual property that encourages the open source community and adopts key standards over time. Hyperledger fabric is one of the blockchain projects in Hyperledger. Like other blockchain technologies, it has books and a system in which participants manage transactions using a smart contract called Chain code. Hyperledger fabric is a platform for distributed ledger solutions based on a modular architecture that provides high confidentiality, resiliency, flexibility and scalability. It is designed to support pluggable implementations of various components and to accommodate the complexities that exist throughout the economic ecosystem. The Hyperledger fabric provides a uniquely elastic and scalable architecture that distinguishes it from alternative blockchain solutions. Planning for the enterprise blockchain of the future requires building a fully validated open source architecture, which is the starting point for Hyperledger. Hyperledger fabric also offers several plug options. Ledger data can be stored in multiple formats and other MSPs are supported by switching consensus mechanisms. In the following, we will examine the function of Hyperledger fabric.

\section{B. Chain code}

The chain code plays the same role as the smart contract function of Ethereum of Hyperledger fabric. A chain code is an asset or software that defines an asset and trading instructions for making modifications. That is, business logic, where chain code enforces rules for reading or changing key value pairs or other state database information. Chain code execution results in the writing of a set of key values that can be submitted to the network and applied to all peer ledgers.

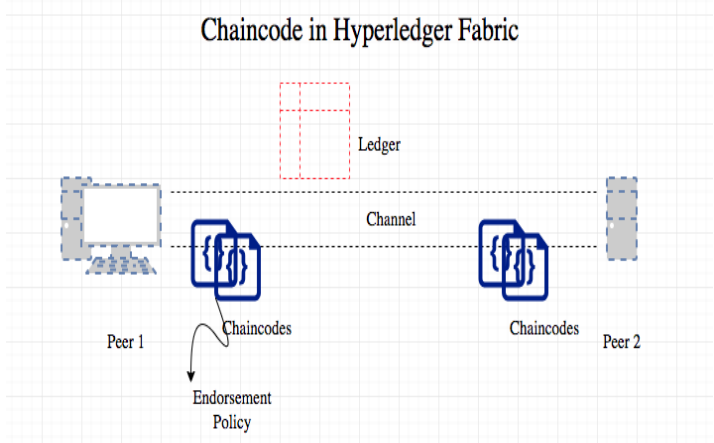

Fig. 1. Chaincode in Hyperledger Fabric

\section{Membership Service Provider(MSP)}

Membership Service Provider (hereinafter referred to as MSP) refers to an abstract component of the system that provides credentials to clients and allows peers to participate in the Hyperledger fabric network. The client uses the credentials to authenticate the transaction, and the peer uses these credentials to authenticate the transaction processing result. Although strongly connected to the transaction processing component of the system, the interface aims to define a membership service component that can implement it without modifying the core of the system's processing component.

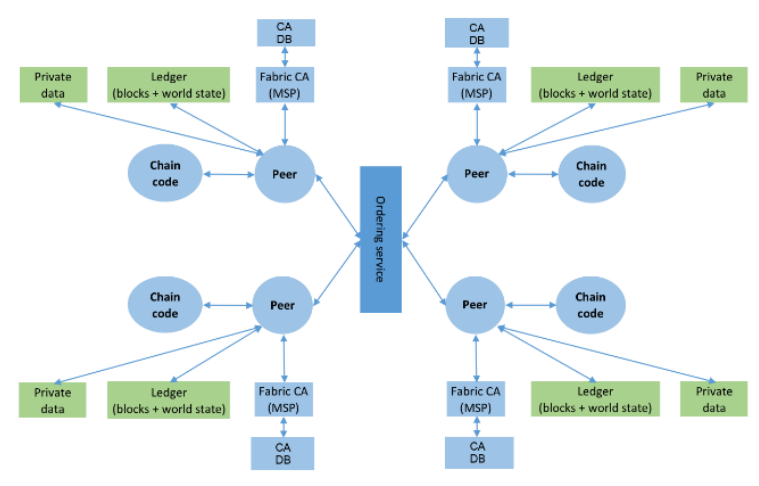

Fig. 2. Configuration of Ordering Service

\section{USed Trading Platform}

\section{A. Background}

Second-hand trade damages are constantly occurring in second-hand trade, which is growing rapidly. For this reason, cases of identity theft frequently occur, and the number of cases of damage to others is increased by exploiting the characteristics 
of non-face-to-face online transactions. In order to prevent such damage, it is necessary to prove the identity by using Hyperledger fabric, make it impossible to forge posts and transaction details by using chain code, identify the criminal's identity by checking the relevant node in case of criminal activity, and then respond promptly. Can be. In addition, the identity of the members is secured using the characteristics of the Hyperledger fabric, and the trader's IP and location data are automatically stored using the chain code.

\section{B. Benefit}

Expected effects can be expressed in two ways. Economically and technically, the blockchain, which cannot be forged and modified, can improve the reliability and increase the trading volume. Can be. Technically, blockchain is used to enhance security, and Hyperledger fabric is used to strengthen membership management to prevent crime. Also, forgery is impossible through chain code.

\section{Consensus Algorithm}

The consensus algorithm is described based on the figure below for quick understanding.

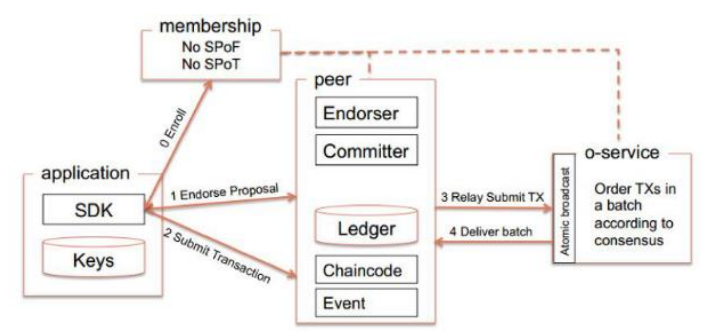

Fig. 3. Consensus Algorithm Configuration

The client offers a deal. The endorsement of the proposed transaction is executed by the chain of peers and the result of execution is signed by RWSet. 'Here, RWSet refers to Read set and Read set. The client submits a transaction after receiving all the results from the guarantee peer according to the guarantee policy. The order service defines the transaction order and creates one block. Confirm before each peer commits a transaction. The peer then checks that there is no conflict between the conditions of the guarantee policy and the transaction.

\section{Processing system}

The processing system is also described with reference to the figure below.

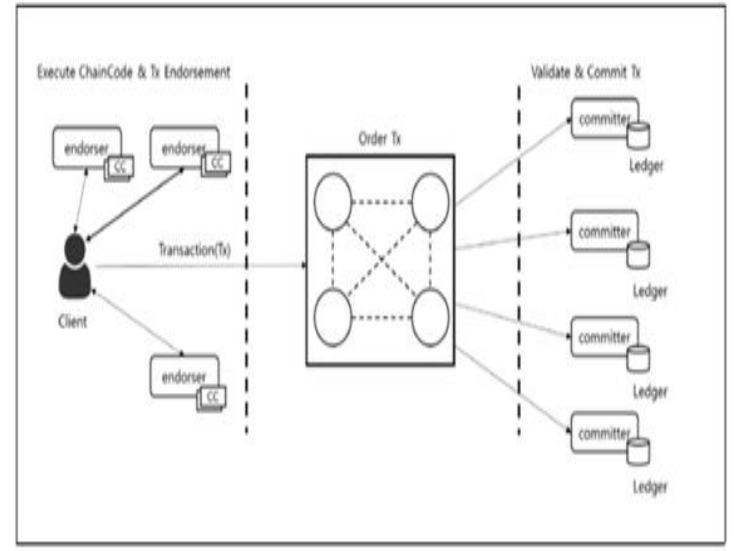

Fig. 4. Execute ChainCode \& Tx Endorsement

The client receiving the execution result transmits the transaction result through the ordering service channel. Order the transactions that occurred earlier according to a consensus algorithm that applies the node that performs the ordering service. Ordered transactions are delivered in a block to all peers connected to the channel. Peers receiving the transaction result confirm the transaction result by confirming that the transaction result is made in accordance with the guarantee policy, and confirm the transaction by storing the result of the appropriately executed transaction in the blockchain.

\section{E. Dynamo DB}

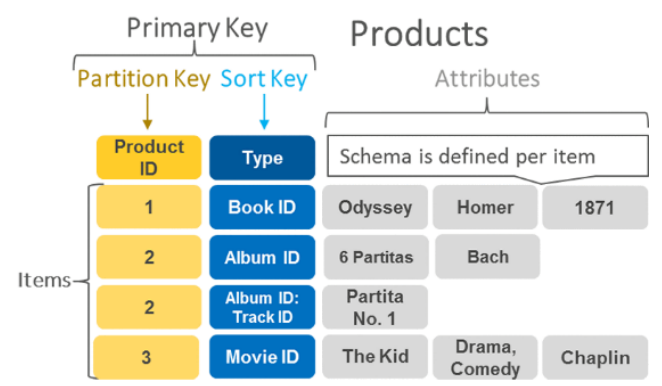

Fig. 5. Configuration of Dynamo DB

Dynamo DB is a fully managed NoSQL DB service that provides fast predictive performance through seamless scalability. Data storage, retrieval, and high level of request traffic can be handled regardless of the data size. On-demand backup function can be used to create a full table backup and maintain it for a long time. It also has high availability and data durability, and can handle events in real time.

\section{F. Algorithm Diagram}

The algorithm diagram is as follows, and the 
detailed explanation is given in order based on the figure.

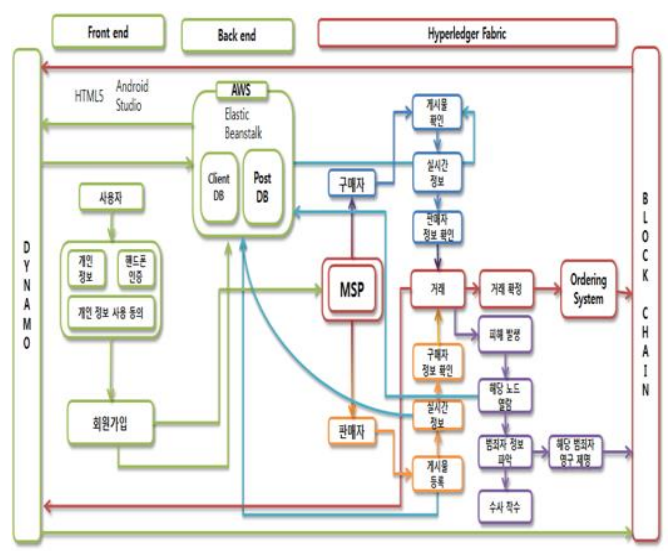

Fig. 6. Algorithm Diagran for Dynamo \& Blockchain

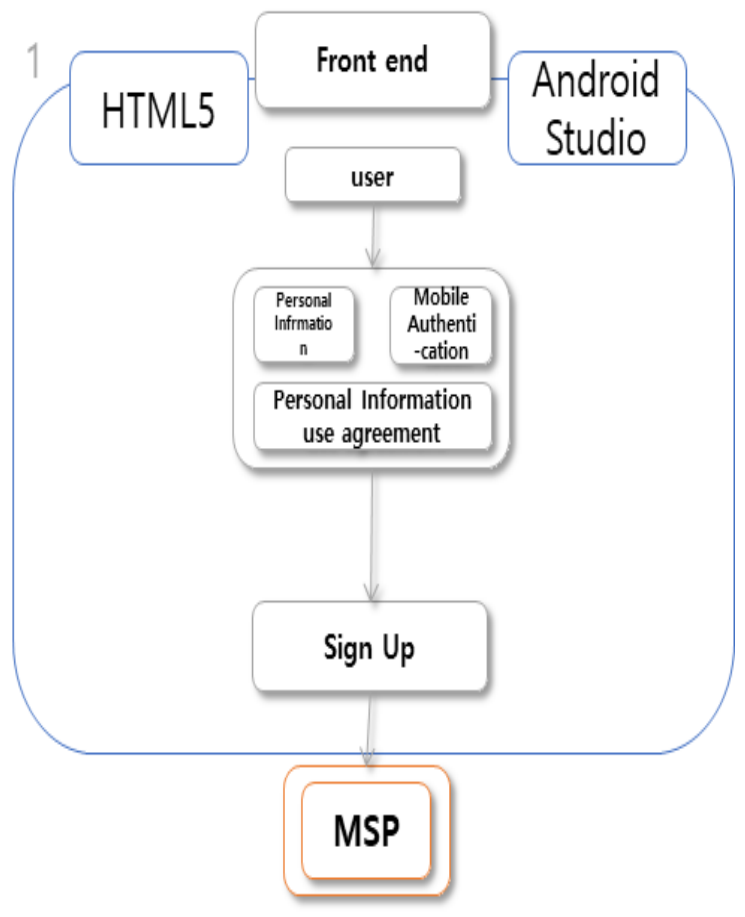

Fig. 7. Relationship between MSP and HTML5

Number 1 is the front end part. The user registers to use the used trading platform. To sign up, fill out the personal information, mobile phone authentication, and personal information agreement, and authenticate your membership through MSP.

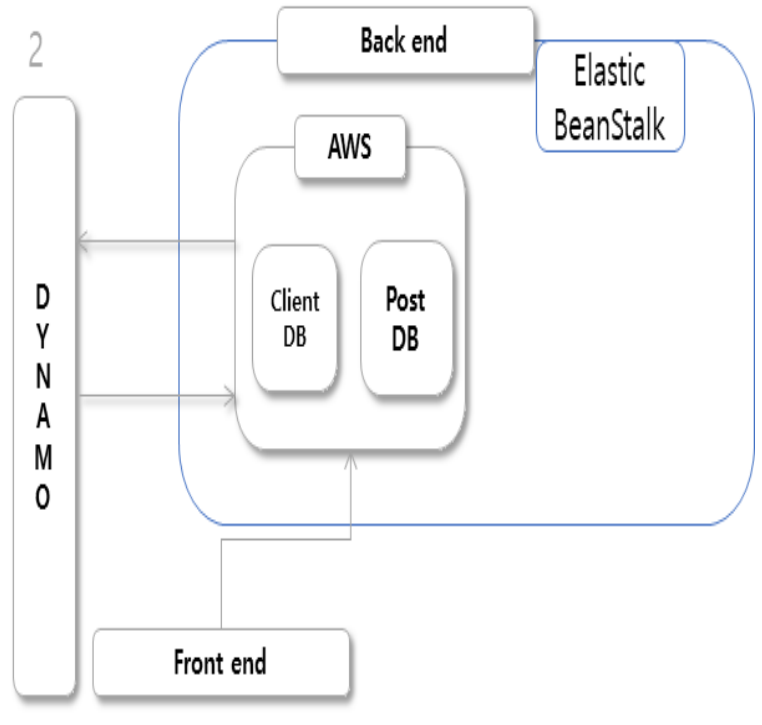

Fig. 8. Configuration of Dynamo

Number 2 is a configuration diagram for storing documents created through membership. Membership information created through membership is stored in Dynamo DB.

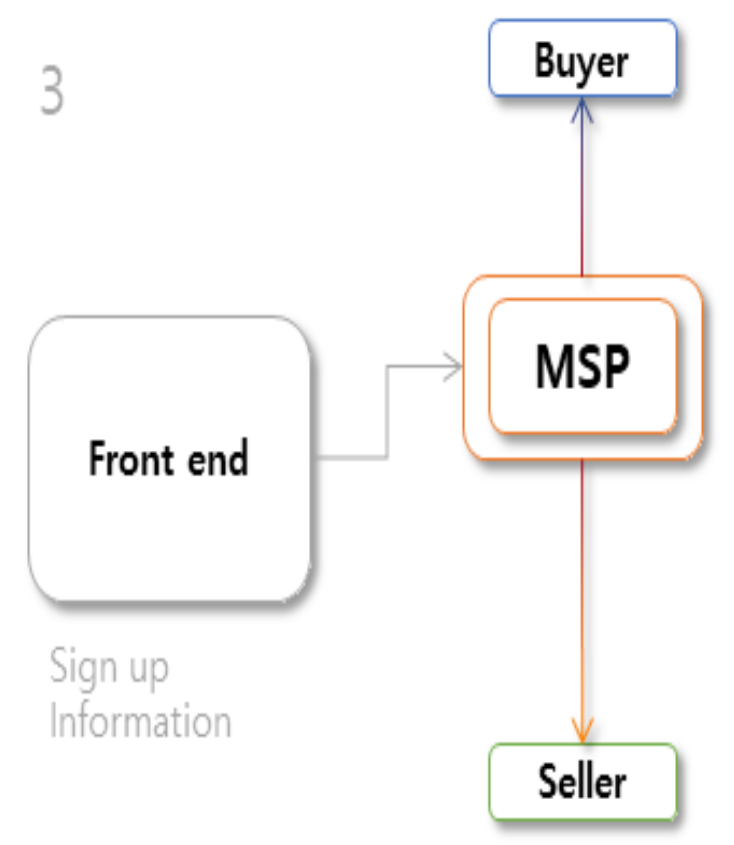

Fig. 9. MSP with Buyer and Seller Configuration

The above figure gives seller and buyer authority to a user who is registered through MSP certification, and can sell as seller authority when selling and buyer authority when purchasing. 


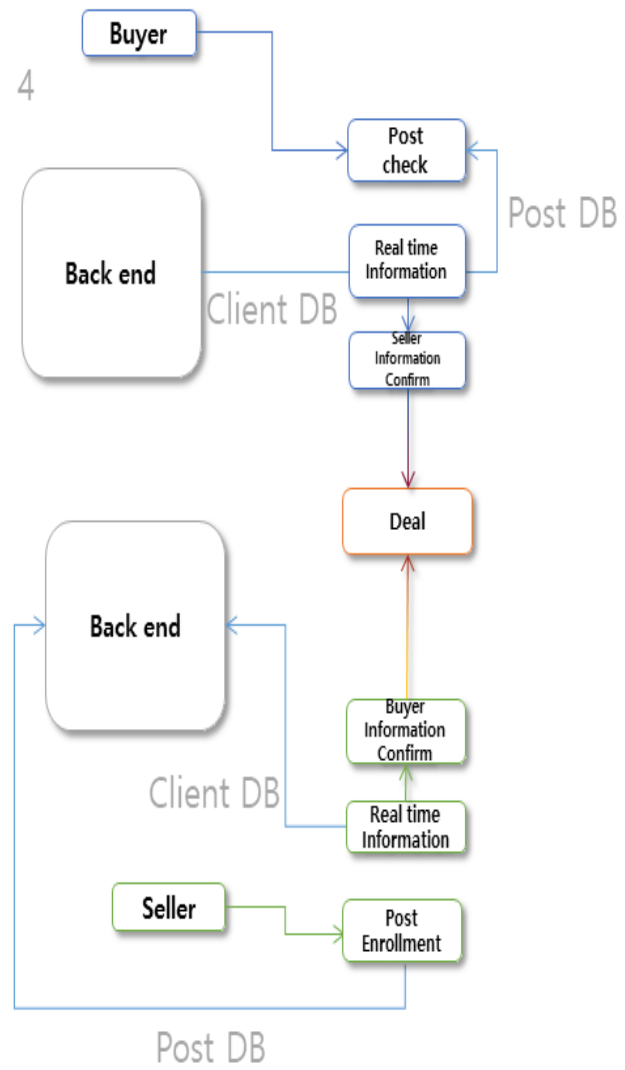

Fig. 10. Configuration of Client DB

The figure above shows the transaction process between seller and buyer. The seller registers the post and the registered post is stored in the Post DB at the back end. The buyer checks the post through the Post $\mathrm{DB}$, and checks the seller's real-time information through the Client DB. The seller and the buyer check each other's information and trade.

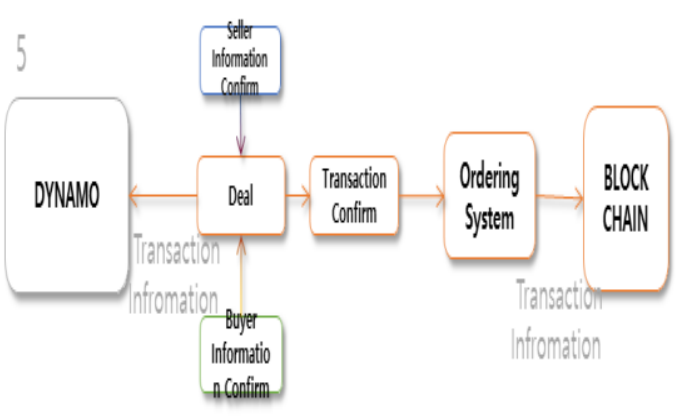

Fig. 11. Flow of Transaction in Dynamo

Transaction information is stored in the DB and block chain, so it cannot be forged.

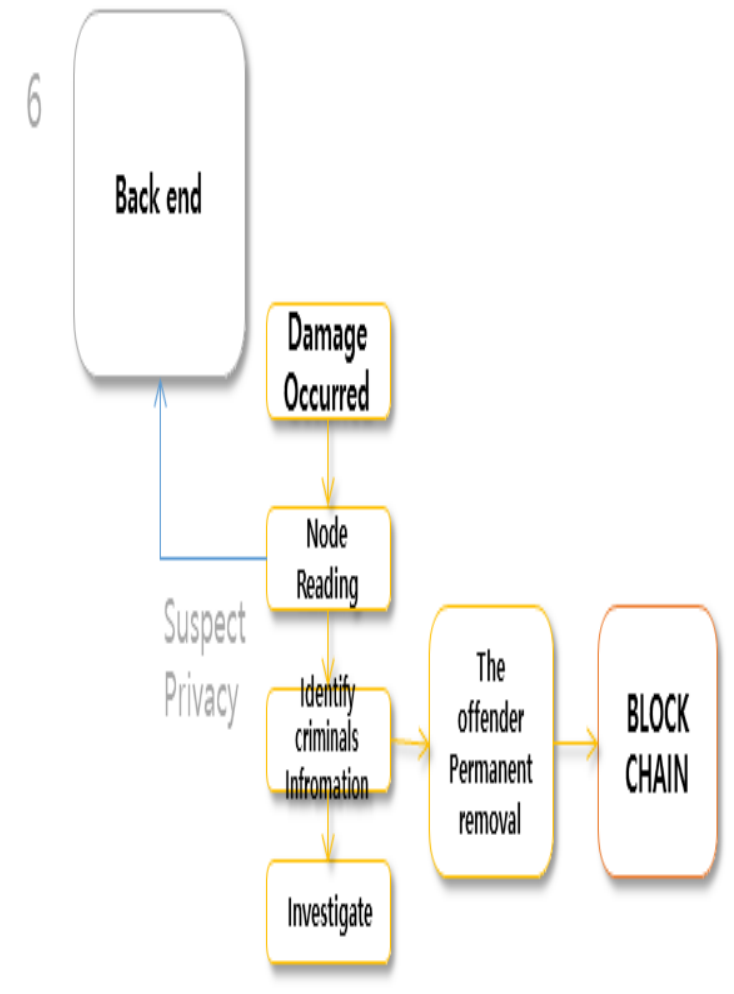

Fig. 12. Flow of Suspect Privacy

In case of damage, criminals are identified by reading the node containing the suspect's personal information stored in the back end. The offender will be permanently expelled from the used trading platform and an investigation will be undertaken using the information.

\section{Conclusion}

Develop a secondhand trading platform using Hyperledger fabric. By using the weakness of anonymity, the disadvantages of the existing used trading platforms where various criminal acts occur, using the impossibility of forgery and alteration which is the characteristic of the blockchain, builds a reliable platform for both sellers and buyers to enhance high satisfaction.

\section{REFERENCES}

[1] Olson, K., Bowman, M., Mitchell, J., Amundson, S., Middleton, D. \& Montgomery, C. (2018). Sawtooth: An Introduction. The Linux Foundation, Jan.

[2] Cachin, C. (2016, July). Architecture of the hyperledger blockchain fabric. In Workshop on distributed cryptocurrencies and consensus ledgers (Vol. 310, p. 4). 
[3] Voell, D. et al. (2016). Hyperledger whitepaper. Published: https://wiki. hyperledger. org/groups/whitepaper/whitepaper-wg.

[4] Nakamoto, S. (2008). Bitcoin: A peer-to-peer electronic cash system.

[5] Sunghyuck Hong (2017). Development of a Secure and Intelligent IoT System based on a Consortium Blockchain. International Journal of Emerging Multidisciplinary Research, 1(2), 9-14. DOI: 10.22662/IJEMR.2017.1.2.009.

[6] Grolinger, K., Higashino, W. A., Tiwari, A. \& Capretz, M. A. (2013). Data management in cloud environments: NoSQL and NewSQL data stores. Journal of Cloud Computing: advances, systems and applications, 2(1), 22.

[7] Schaefer, C., \& Edman, C. (2019). Transparent Logging with Hyperledger Fabric. 2019 IEEE International Conference on Blockchain and Cryptocurrency (ICBC).

[8] Huh, S., Cho, S. \& Kim, S. (2017, February). Managing IoT devices using blockchain platform. In 2017 19th international conference on advanced communication technology (ICACT) (pp. 464-467). IEEE.

[9] Sousa, J., Bessani, A. \& Vukolic, M. (2018, June). A byzantine fault-tolerant ordering service for the hyperledger fabric blockchain platform. In 2018 48th annual IEEE/IFIP international conference on dependable systems and networks (DSN) (pp. 51-58). IEEE.

[10] K. H. Lee. (2018). A Study on the type of BlockChain (Ethereum, Hyperledger Fabric), Proceedings of the Korean Institute of Communication Sciences, 442-443. 